\title{
An Overview of Alkylresorcinols Biological Properties and Effects
}

\author{
Anastasia A. Zabolotneva $\mathbb{D},{ }^{1}$ Olga P. Shatova $\mathbb{D},,^{1}$ Anastasia A. Sadova $\mathbb{D},^{1}$ \\ Aleksandr V. Shestopalov $\mathbb{D}^{1}{ }^{1}$ and Sergei A. Roumiantsev $\mathbb{D}^{2}$ \\ ${ }^{1}$ N. I. Pirogov Russian National Research Medical University, 1 Ostrovitianov str., 117997 Moscow, Russia \\ ${ }^{2}$ Center for Digital and Translational Biomedicine, Center for Molecular Health, 32 Nakhimovskiy Prospekt, \\ 117218 Moscow, Russia \\ Correspondence should be addressed to Anastasia A. Zabolotneva; a.zabolotneva@gmail.com
}

Received 30 September 2021; Revised 11 December 2021; Accepted 14 December 2021; Published 5 January 2022

Academic Editor: José María Huerta

Copyright (C) 2022 Anastasia A. Zabolotneva et al. This is an open access article distributed under the Creative Commons Attribution License, which permits unrestricted use, distribution, and reproduction in any medium, provided the original work is properly cited.

\begin{abstract}
The investigation of alkylresorcinols has drawn an increasing interest recently. Alkylresorcinols (ARs) are natural chemical compounds synthesized by bacteria, fungi, sponges, and higher plants, possessing a lipophilic polyphenol structures and a myriad of biological properties. Human takes ARs as a component of a whole grain diet (from whole grain rye, wheat, and barley products), and thus, alkylresorcinols are frequently used as whole grain intake markers. Besides, ARs are considered as promising bioregulators of metabolic and immune processes, as well as adjuvant therapeutic agents for antimicrobial and anticancer treatment. In this review, we attempted to systematize the accumulated information concerning ARs origin, metabolism, biological properties, and their effect on human health.
\end{abstract}

\section{Introduction}

The interest in the investigation of the biologically active components of grain products has been progressively rising for the past few years due to the intriguing recent observations that a whole grain diet is strongly correlated with the lower risk of obesity, diabetes, and some cancers, namely, breast, colon, and prostate cancer [1] and that a gluten-free diet is inversely associated with type 2 diabetes development [2]. Besides, in the last decade it has become clear that not only diet, but also the human gut microbiome provide a lot of regulatory molecules that influence host metabolism and immunity functioning directly or indirectly [3] through the formation of interconnections between the gut and adipose tissue [4], the gut and nervous system [5], the gut and immune system [6], etc. In this regard, the investigation of alkylresorcinols is of particular interest.

ARs are naturally synthesized by plants (like rye, wheat, and barley), fungi, and bacteria and possess lipophilic polyphenol structures (Figure 1) along with many biological activities. Type III polyketide synthases (PKSs) found in all domains of life are in charge of the synthesis of $\mathrm{AR}$ as well as other aromatic polyketides [7]. Interestingly, srs-like operon in prokaryotes, which contains $s r s A$ gene (Streptomyces resorcinol synthase A) encoding PKS type III, is commonly found among Gram-positive and Gramnegative bacteria (including Actinobacteria, Cyanobacteria, Proteobacteria, and Halobacteria) [8]. This fact indicates that phenolic lipids are widely distributed among the organisms of this domain of life. In bacteria, ARs are the components of plasma membranes, which may increase their rigidity and thus protect cells from dangerous compounds like antibiotics or from unfavorable environmental conditions. However, the actual functions of ARs found in different organisms remain to be elucidated and are currently under investigation. Herein, we aimed to observe ARs biological properties known to date and to review their effects on human metabolism in link with chronic disorders.

\section{The Structure and Synthesis of ARs}

As mentioned above, ARs are lipophilic molecules with polyphenol structure (Figure 1), which exhibit various biological functions and as many different aromatic polyketides are synthesized by type III PKSs [7]. 
<smiles>[R]c1cc(O)cc(O)c1</smiles>

FIGURE 1: General structure of ARs. R-alkyl or alkenyl side chain, oxygenated $\mathrm{R}$ is also possible. The $\mathrm{R}$ position may be changed and presence of additional polyphenolic rings is possible depending on the type of organism synthesizing ARs.

In the reactions catalyzed by these enzymes, a stater substrate such as acyl-CoA and an extender substrate such as malonyl-CoA are iteratively decarboxylated. Following the condensation reaction, the resultant polyketide chain is then cyclized to yield dissimilar bioactive natural compounds dramatically diverse in their structure. This structural variety of polyketide scaffolds is governed mainly by the enzyme's selectivity to starter and extender substrates, the number of condensation reactions, and the mode of ring closure of the resultant polyketide chains [9] (Figure 2).

\section{The Natural Sources of ARs and Their Absorption}

On the first glance, it seems that the only source of ARs in the human diet is the outer layers of wheat and rye kernels where they are present in significant amounts [11]. At the same time, ARs were found in mango peels, leaves, and seeds [12]. Besides, alkylresorcinols were shown to be one of the main components in whole grain products possessing marked antiproliferative properties against the growth of cancer cells in humans [13]. Additionally, ARs may enter the human body as preservative agents (particularly, 4-hexylresorcinol) found not only in food, but also in several medications, toothpaste, and cosmetics [14]. Interestingly, the human gut microbiome can be considered as a source of ARs as well. Prokaryotes (namely, Actinobacteria, alpha-, delta-, and gamma-Proteobacteria, and Cyanobacteria) are able to synthesize different monoalkylresorcinols [15-18] which demonstrate anticancer, antifungal, anti-inflammatory, antimicrobial, antiparasitics, antioxidant, and genotoxic activities [19]. It is worth to note that, in the study of Martins et al., a phylogenetic analysis of the genes coding for type III PKS enzymes revealed a wide abundance of such sequences among different members of Cyanobacteria phylum [18]. In addition, the presence of srs-like operon structures coding for type III PKS in numerous Gram-positive and Gramnegative bacterial species was reported in the study of Funabashi et al. [8]. Taken together, these data demonstrate that the ability to synthesize ARs is common to many prokaryotes. Thus, one should not exclude the bacterial origin of ARs found in human biological samples, although, to date, there is no unambiguous evidence that the human microbiome contributes to the body pool of ARs.

Intriguingly, small concentrations of ARs, namely, 3,5dihydroxybenzoic acid (DHBA) and 3-(3,5-dihydroxyphenyl)-propanoic acid (DHPPA), were found circulating in the blood of volunteers abstained from consuming the whole grain wheat and rye for one week [20]. The small

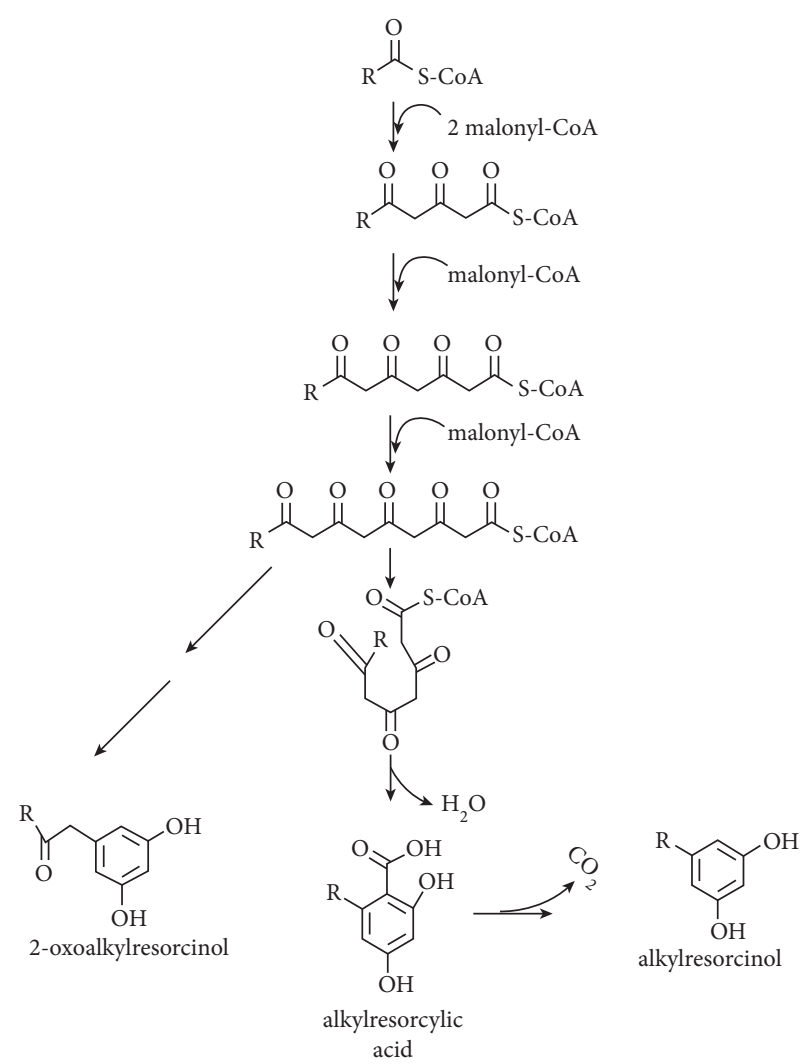

FIgURE 2: A proposed biosynthetic pathway of ARs by PKSIII according to [10].

concentrations of these compounds could arise from accidental consumption of cereal products containing minor amounts of ARs or liberation of ARs from stored body pools [21], or they could be produced by the gut microbiome. However, according to the authors, ARs with long side chains (C15-C25), that are likely to have plant origin, predominantly circulated in plasma (Figure 3 ).

Unfortunately, no researches evaluating the concentration of other types of ARs, including those produced by microbes, have been conducted so far.

The absorption of ARs is commonly investigated using an ileostomy model $[22,23]$. The observations in pigs, rats, and humans demonstrate that the concentration of ARs in plasma rises proportionally to the intake of ARs as a food component, and similar results were shown for different model organisms [24]. At the same time, only $50-70 \%$ of the consumed ARs are absorbed [25]. The absorption of ARs occurs apparently in the small intestine by passive diffusion and may also happen similarly to tocols with the help of active transport provided by scavenger receptor class B (SRBI) [26]. Next, enterocytes pack ARs into chylomicrons and release them to the lymphatic system. Another proposed way of ARs absorption concerns the direct transfer of these molecules to high-density lipoproteins in the intestine and their subsequent distribution into blood lipoproteins [27]. Thus, the major fraction of ARs in plasma is included in different lipoprotein (LP) fractions, namely, very low-density LP (VLDL), high-density LP (HDL) and low-density LP 


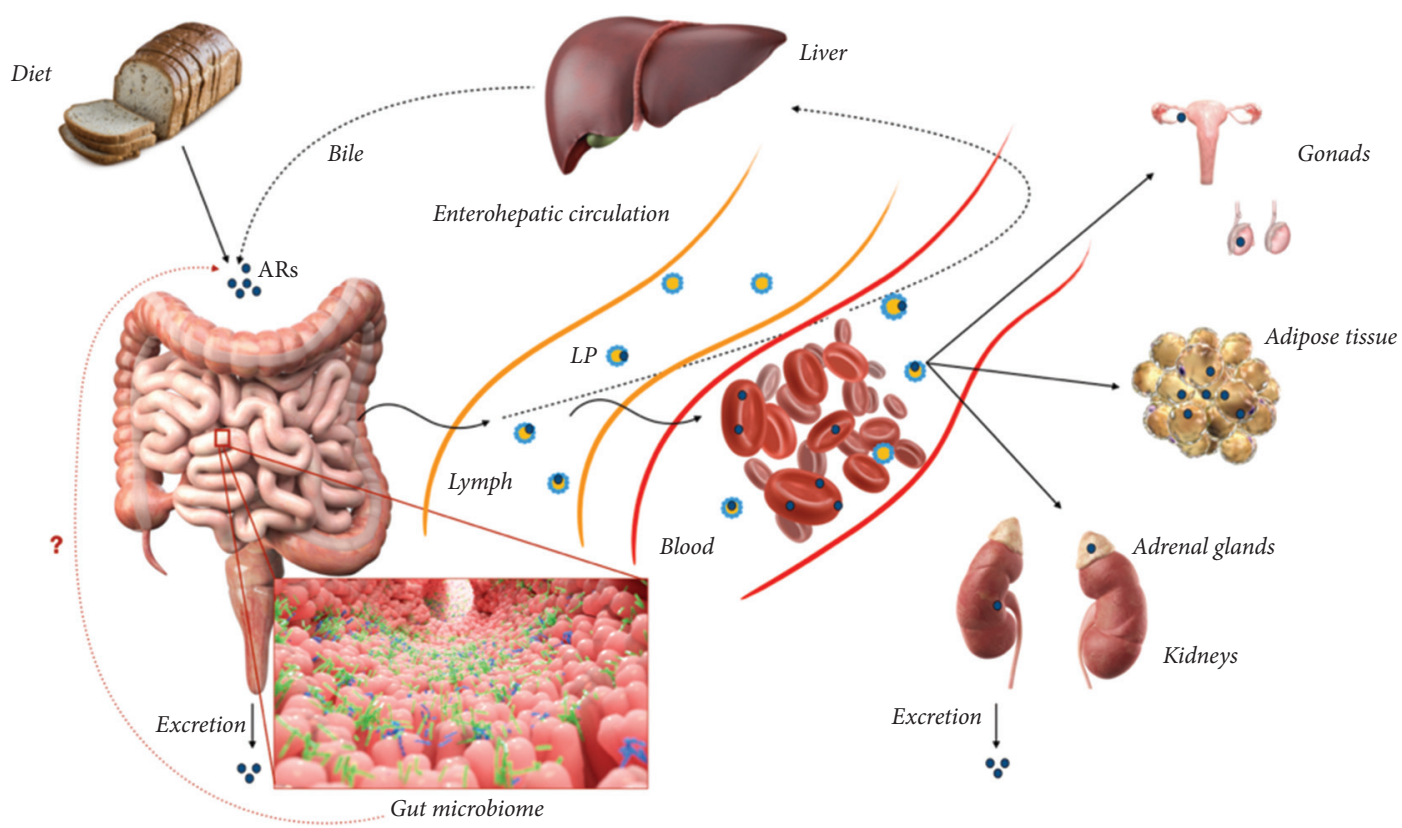

FIgURE 3: ARs absorption and metabolism. ARs enter the human body mainly with whole grain products, although gut microbiome origin of ARs is also possible. ARs are transported through the lymph and blood as a part of lipoproteins (LP) toward the liver and other tissues. ARs are metabolized in the liver and excreted through the kidneys with urine or through the intestine with feces.

(LDL), while none of ARs are present in the water fraction of blood plasma. Indeed, VLDL and HDL are considered the main carriers of ARs in fasting samples (Figure 3).

Further pathways of ARs utilization in the body embrace their distribution to the membranes of red blood cells as well as their transport to hepatocytes and different cells and tissues. Interestingly, ARs may shorten the erythrocytes lifespan by destabilization of their membranes, and this haemolytic activity of ARs homologs depends on the degree of side chain unsaturation and is inversely proportional to the chain length [28].

Based on the data reported above, ARs and their metabolites, namely, DHBA and DHPPA, seem to be promising biomarkers of whole grain consumption [22]. Similarly, the rising of ARs levels in blood may serve as the evidence of the breach of a gluten-free diet. Finally, the study of ARs and their homologs will be of particular importance in the investigation of gluten effects in the wider population [29].

\section{ARs Metabolism}

The metabolism of ARs in the liver consists of two phases and is similar to the oxidation of tocopherols. The first step results in the introduction of a hydroxyl group at the end of the alkyl tail of the molecule in the $\omega$-oxidation reaction catalyzed by CYP4F2 (Figure 4). Next, the hydroxyl group is oxidized to a carboxylic acid followed by $\beta$-oxidation. ARs alkyl chain is shortened in this way resulting in two main metabolites, DHPPA and DHBA, which are water soluble molecules. During the second phase, the conjugation of the reaction products (ARs, their intermediates, and final metabolites) with glucuronide sulfate groups and amino acids happens, which significantly improve the urinary excretion of the molecules. Since the two AR metabolites, DHPPA and
DHBA, can be detected in both human plasma and urine [30], they can be considered as biomarkers of whole grain wheat and rye intake [31] (Figure 4).

The application of radiolabeled ARs in the investigation of ARs metabolism in animals demonstrated that they are accumulated in the body in insignificant amounts [32]. However, in case of prolonged feeding with ARs, they are stockpiled in adipocytes and blood cells, as it was demonstrated in rats [23]. In addition, the body pool of ARs may include those molecules incorporated into the membranes of lipid transport vesicles or associated with plasma proteins [33].

\section{The Biological Effects of ARs}

ARs have been studied using in vitro experiments and metaanalysis, which have revealed a wide range of bioactivities exhibited by these molecules, namely, antimicrobial, anticancer, antilipidemic, antioxidant, and others described below.

5.1. ARs Are Autoregulators in Microbial Communities. In a population of bacteria, one can identify vegetative or actively proliferating cells and the dormant ones, which enter the quiescent state and do not divide. These dormant cells form metabolically inactive endospores, which are in charge of the establishment of persister cells in the given population. Endospores are characterized by high resistance to such unfavorable environmental conditions as radiation and high temperature, as well as by tolerance to antibiotics and antimicrobial chemicals [34]. Thus, the emergence of persister cells is one of the mechanisms of antibiotic resistance development leading to chronic bacterial infections and 


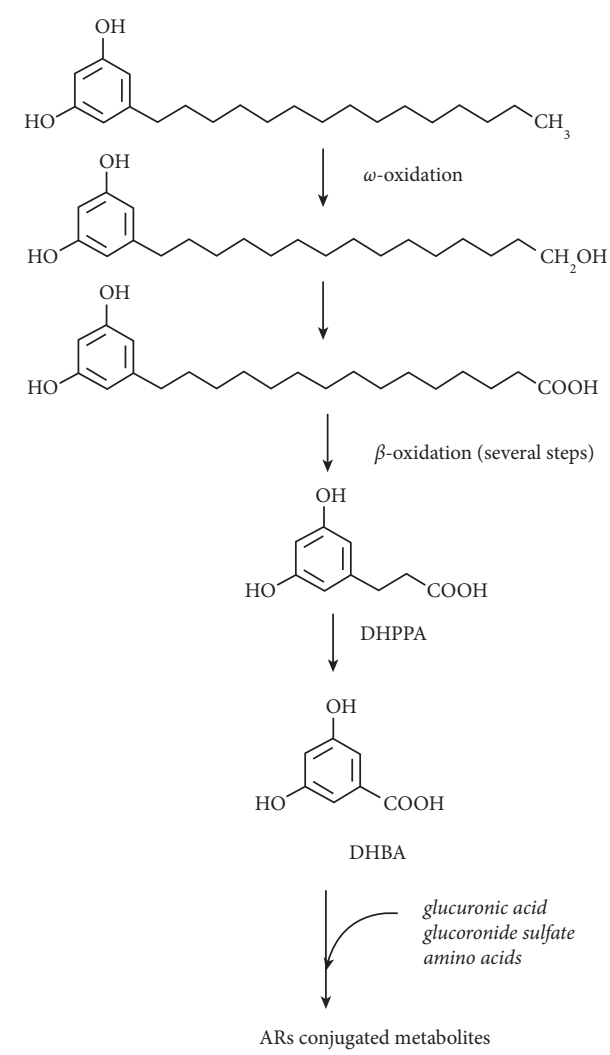

FIGURE 4: ARs metabolism in mammalian cells (pentadecylresorcinol (C15:0) is shown as an example). Hydroxyl groups on the phenolic ring are conjugated with amino acids, glucuronide, and/or sulfate groups during metabolism.

metabolic shifts in host organisms [35-37]. In this regard, it is of particular interest that ARs (namely, 4-hexylresorcinol) in combination with antibiotics were shown to dramatically decrease the number of germinating $B$. cereus spores both in liquid medium and on agar plates compared to the treatment with antibiotics alone [34]. These results are consistent with the data from other studies dedicated to the investigation of ARs autoregulation potential [38]. We propose that ARs entering the human body with food products should be considered as autoregulators in the gut microbial community, which may influence the regulatory axes "gut-immune system», «gut-nervous system», "gut-adipose tissue».

5.2. Antimicrobial Properties of ARs. Nowadays, clinical practice faces the problem of increasing antibiotic resistance of pathogenic bacteria. In an attempt to address this issue, two approaches have been developed, the first being the search for new powerful antimicrobial agents, and the other being the use of antibiotic adjuvants capable of enhancing the antimicrobial activity of the commonly used chemicals. For instance, Nikolaev et al. [34] tested the combination of ARs with 12 clinically used antibiotics against a broad range of pro- and eukaryotic pathogenic microorganisms. They found the application of 4-hexylresorcinol as an adjuvant to polymyxin to be highly efficient against the germination of bacterial dormant forms (spores) and to prevent the formation of antibiotic-resistant persister cells. The stunning results were obtained with the use of an in vivo mouse model of K. pneumoniae-induced sepsis: $75 \%$ of the animals recovered from the infection after treatment with the combination of ARs and the antibiotic, in contrast to the group receiving the antibiotic alone, in which none of the animals were cured. Thus, the authors conclude that ARs such as 4hexylresorcinol can be used as an adjuvant to increase the efficiency of several known antibiotics and to decrease the risk of antibiotic resistance development.

ARs display antimicrobial activity by incorporation into the cell walls of microorganisms. Gram-positive bacteria possessing peptidoglycan-based outer cell wall were shown to be highly sensitive to ARs treatment. On the contrary, Gram-negative bacteria, with their additional outer lipid membrane, and yeast and other fungi with complex chitin and polysaccharide-based cell walls seem to resist ARs insertion into their membranes. Besides, the presence of lipid structures and mycolic acid makes the cell surface more hydrophobic, which is a strong obstacle for ARs penetration. According to the published data, the antimicrobial activity of n-alkylphenols is higher in those with longer alkyl chains, but the enrichment of microbial cell walls by lipids interferes with the ARs activity against microorganisms [19]. They suggest that the biological activity of ARs is likely governed by their ability to act as structural modifiers of biopolymers and supramolecular structures such as membranes $[12,19,39,40]$. Presumably, ARs increase the membrane viscosity by association with lipid molecules and macromolecules within membranes, inhibiting their functional activities. Additionally, a dose-dependent interaction of ARs with DNA and proteins was reported, which is mediated by the formation of reactive oxygen species (ROS) and by the activation of RpoS (RNA polymerase, sigma factor S) in case of the increase in the concentration of ARs within the cell [41]. Based on these facts, it seems unlikely that ARs may cause microbial resistance, since their action is nonspecific and extends from bacteria to protozoans and helminths [19].

5.3. ARs Influences Gut Microbiome Composition. Gut microbiota not only closely interacts with dietary products, but is also interconnected with genetic factors, being one of the important causes of metabolic disorders and obesity development. However, the mechanisms underlying the influence of gut microbiota on host metabolism remain to be elucidated. Studies in animals and humans have demonstrated a strong correlation between the alteration of the microbial composition in the intestine, manifested in the shift toward increased energy harvest, and the obese phenotype [42, 43]. Additionally, the increasing ratios of Firmicutes (such as C. coccoides, C. leptum, and Enterococcus) to Bacteroidetes (such as Bacteroides and Prevotella) within the microbial community of the gut were reported to be associated with obesity and metabolic disorders [44]. As a whole grain diet was demonstrated to have prebiotic effects [45], the study in which the fecal microbiota in mice supplemented with ARs were examined is of particular interest [46]. Oishi et al. managed to show that food ARs 
significantly increased the amount of Prevotella and reduced the amount of Enterococcus in mice feces. Additionally, the fecal amount of the latter bacteria positively correlated with body weight and fecal bile acids but negatively correlated with the fecal amount of total lipids. It was suggested that the metabolic improvements demonstrated in this study were stimulated by ARs consumption, which thus manifested their prebiotic effects. These data are in concordance with the results of the investigation of antiobesity effects of prebiotic supplementation in humans and other animals [45]. It is also reported that polyphenols from beverages, fruits, and vegetables influence, i.e., may stimulate or prevent, the growth of bacteria in the gut [47].

On the other hand, in a randomized, controlled crossover trial comprising two 8-week dietary intervention periods in 60 Danish adults exhibiting an increased metabolic risk profile, it was demonstrated that a whole grain diet, compared with a refined grain diet, led to weight loss and to the decrease of low-grade systemic inflammation markers without significantly changing the fecal microbial composition, diversity, or functional potential [24]. It is important to note that the effect of whole grains on inflammation markers was still evident after adjustment for weight loss. Interestingly, the concentration of the proinflammatory cytokine interleukin-6 (IL-6) in fasting serum was lowered in response to the intake of ARs $\mathrm{C} 17: 0$, rye products being the main source of it [24].

However, the consumption of whole grains for 8 weeks did not affect much either glucose or lipid metabolism; liver fibrosis markers, metabolic satiety-regulating hormones, or blood pressure did not show any changes in the tested individuals as well compared with refined grain intake. Thus, one may speculate that the body weight of the trial participants supplemented with whole grain products was reduced mainly because the decreased energy intake for this type of food leads to satiation faster in comparison with refined grain products. Moreover, according to this study, the whole grain diet did not influence gut permeability [24].

At the same time, the fact that the amount of proinflammatory markers circulating in the blood is strongly inversely correlated with whole grain biomarkers, such as ARs, suggests a great potential of alkylresorcinols as healthpromoting agents.

Contradictory results of the studies described above may result from different methods and approaches of microbiome analysis as well as from the diversity in metabolic characteristics of the investigated populations. Nevertheless, whole grains may be considered as a part of a healthy diet providing the normalization of body weight and reduction of systemic inflammation.

\subsection{ARs Regulate Sirtuin (SIRT) Activity and Show Antiaging} Potential. Restriction in calorie intake has been associated with the prolonged lifespan, and despite the fact that this phenomenon was confirmed in multiple epidemiological studies and different animal models, its underlying mechanisms have not yet been revealed [48]. To date, the observations associate the reduced glucose concentration in cell growth medium with the increased expression of sirtuins, particularly SIRT1 [49]. Sirtuins (SIRTs) belong to a class of proteins with NAD+-dependent deacetylase or adenosine diphosphate-ribosyltransferase activity and have been shown to participate in diverse cell functions such as DNA repair and recombination, gene silencing, senescence, and responses to stressors [50]. ARs were demonstrated to modulate the activity of SIRT1 by affecting the enzyme structure, most likely by allosteric activation of the enzyme, with no effect on the SIRT1 expression level [51]. According to this study, ARs are able to activate sirtuins in mammalian cells, as it was demonstrated that the percentage of histone acetylation in the human monocyte cell line THP-1 significantly decreased in response to C17 AR. Mediated by another SIRT family member, namely, Sir2, ARs were shown to prolong $D$. melanogaster lifespan in conditions of restricted calories intake [51]. Among other things, ARs addition to the 3T3-L1 cell culture prevented triglyceride accumulation in cells, indicating ARs potential to block triglyceride synthesis in vivo [52]. Numerous studies demonstrate that ARs alter the metabolism of lipids in a SIRTmediated pathway, likely to forestall the development of metabolic syndrome [51].

5.5. ARs and Wound Healing. It has been known for decades that certain ARs possess antiseptic properties along with a broad-spectrum antimicrobial activity [53, 54]. Thus, 4hexylresorcinol was demonstrated to significantly decrease the expression of tumor necrosis factor $\alpha$ (TNF- $\alpha$ ) in RAW264.7 cells and in a rat burn wound model [55]. Concurrent with TNF- $\alpha$ suppression, 4-hexylresorcinol also induced rapid epithelization and collagen regeneration in animals, compared to the control group. These beneficial properties of hexylresorcinol have been successfully applied in many cosmetics products with antiaging and lightening effects $[56,57]$.

5.6. ARs May Prevent Muscle Atrophy. A decline in muscle mass, termed muscle atrophy, occurs under conditions of muscle disuse (e.g., immobilization, denervation, and muscle unloading), fasting, and aging and accompanies such diseases as cancer cachexia, Cushing's syndrome, sepsis, diabetes mellitus, and chronic renal failure [58]. From the point of view of biochemistry, muscle atrophy can be caused by decreased protein synthesis and/or increased protein decay [59]. Aging- and inactivity-mediated loss of muscle strength is associated with the accumulation of intramuscular triglycerides with the simultaneous progressive decrease in the mass of muscle $[60,61]$. Hiramoto et al. reported the effects of ARs on denervation-induced muscle atrophy studied in mouse models [59]. According to this investigation, ARs supplement prevented the reduction of myofibers and their mass in skeletal muscle caused by denervation, although ARs failed to suppress the upregulated gene expression of muscle atrophy associated ubiquitin ligases and autophagy-associated genes. The expression of p62, considered as a marker of autophagy, was thus higher in the atrophied muscle of mice fed with ARs-supplemented 
diet. Based on these results, the authors proposed that energy metabolism in the atrophic muscle of mice provided with ARs was altered from the oxidation of glucose to the oxidation of fatty acids instead, which prevented the infiltration of muscle tissue with fat and protected it from autophagy [59].

5.7. ARs Exhibit Neuroprotective Potential. Gradual reduction of cell number in certain populations of neurons in the central nervous system results in the development of neurodegenerative disorders (ND) [62], which mostly affect elderly people and manifest in cognition or memory impairments leading to the individual's death [63]. In the light of this, the search for efficient neuroprotective agents is an issue of the day. Phenolic compounds, considered as multitarget drugs, are promising factors able to prevent oxidative stress and modulate the activity of endogenous enzymes and receptors associated with neuroprotection. As it was shown recently, wheat bran-originated ARs demonstrated neuroprotective effects in $\mathrm{H} 2 \mathrm{O} 2$-treated HT22 cells through acting as antioxidants and controlling the Nrf2ARE pathway [64]. The latter is known to play a crucial role in cellular protection upon exposure to oxidative stress. The mouse immortalized neuronal HT22 cells derived from the hippocampus, one of the key brain structures in charge of memory formation [65], are frequently used as an in vitro model to investigate the mechanisms of neuronal cell death and neurodegenerative diseases [66]. Oxidative stress in such models is stimulated by $\mathrm{H}_{2} \mathrm{O}_{2}$, which serves as a source of reactive oxygen species (ROS), thus demonstrating neurotoxicity properties and dramatically decreasing cell viability [67]. In the study of Fan et al., the HT22 cells pretreated with ARs before $\mathrm{H}_{2} \mathrm{O}_{2}$ treatment demonstrated a significant dose-dependent increment in viability and ROS decline, indicating that ARs could protect cells from $\mathrm{H} 2 \mathrm{O} 2$ induced oxidative stress [64]. Moreover, the oxidative damage of cells was inhibited by ARs through the activation of the Nrf2/ARE signaling pathway, for the ARs are believed to promote the translocation of Nrf2 to the nucleus and to upregulate its transcription. Following this scenario, the activated nuclear form of Nrf2 is likely to further induce phase II antioxidant enzyme gene transcription [68]. These suggestions explain the marked increase in the expression of HO-1, NQO1, GCLC, and GCLM in HT22 cells in response to ARs. Taken together, these data imply that ARs could be used as an excellent dietary component with the neurodegenerative protective effect to improve human health.

5.8. Anticancer Properties of ARs. Numerous epidemiological studies report the anticancer effect of a whole grain diet against breast, colon, and prostate cancer [25, 69] and, in particular, the ability of wheat bran-originated ARs to block the proliferation of human prostate adenocarcinoma cells and colon cancer cells $[11,69]$. The antitumor mechanism of ARs consists in the destruction of tumor cells' DNA and the prevention of DNA repair in them [70], thus increasing the rate of cell death with genetic toxicity [71], which stops the formation of new cancer cells. As recent investigations indicate, natural plant extracts such as flavonoids and polyphenols are able to stimulate apoptosis- and autophagyassociated intracellular pathways to precipitate the death of tumor cells and to stop their proliferation [69].

In the study of Oskarsson and Ohlsson [72] carried out on the human adrenocortical cell line H295R, the nontoxic concentrations of C15:0 and C19:0 ARs directly affected the steroidogenesis; namely, they reduced the synthesis of testosterone and decreased the secretion of estradiol, cortisol, and aldosterone. The authors proposed that the decline in the secretion of the hormones could appear due to the inhibition of the activity of CYP17 enzyme, which is in charge of the conversion of pregnenolone to $17 \alpha-\mathrm{OH}$ pregnenolone and of progesterone to $17 \mathrm{a}-\mathrm{OH}$-progesterone. The blocked enzyme's activity might result in the shift from glucocorticoid and androgen synthesis toward the synthesis of aldosterone observed in the study. This hypothesis is supported by the fact that, under the treatment of cells with mixtures of ARs with lower concentrations, the decrease in androgen and cortisol secretion accompanied a slight increase in aldosterone production. The results are of particular importance, as CYP17 is considered as a key enzyme in the synthesis of steroid hormones and a key target in the treatment of prostate cancer [73], in which cells depend on androgens needed for their growth. Thus, the inhibition of CYP17 activity by ARs blocking androgen production may be a promising approach in anticancer therapy to reduce the proliferation of prostate cancer cells. Other studies also confirm the association between rye intake or ARs consumption and the probability of oncological disease development. For instance, according to Torfadottir et al. [74], adults who include rye bread in their daily rations have a reduced risk of prostate cancer, particularly, advanced prostate cancer, development. On the other hand, in another investigation they reported no significant protective effects of whole grain consumption and the associated AR metabolites in plasma on incident prostate cancer [75].

Similarly to the correlation between the level of androgens and the risk of prostate cancer, prolonged exposure to estrogens may lead to the development of breast cancer, and more than $60 \%$ of breast cancers are found to be estrogen-dependent [76]. As ARs were shown to decline estradiol secretion, rye product consumption may be recommended as a countermeasure capable of reducing the risk of breast cancer development [77]. Along with ARs leading to decreased plasma estrogen levels, the generally high dietary fiber intake was reported to be associated with the lower risk of breast cancer as well $[78,79]$. Besides the mechanism described earlier, ARs, as highly lipophilic molecules, are proposed to affect steroid hormone production through the accumulation in tissues enriched in lipids, such as adipose tissue, testis, ovaries, and adrenal cortex, which are in charge of steroidogenesis [72].

ARs reliably not only reduces the risk of prostate cancer in men and breast cancer in women, but is also associated with the decreased risk of colorectal cancer development in both genders [80, 81]. Kyro et al. in their study of ARs intake association with colorectal cancer, conducted on a large, multicenter cohort with more than one million individuals 
from 10 European countries, considered the plasma concentrations of different ARs homologs, namely, C17:0, C19: $0, \mathrm{C} 21: 0, \mathrm{C} 23: 0$, and $\mathrm{C} 25: 0$, and the incidence rate ratio (IRR) of distal colon cancer in matched case-control pairs [80]. In participants with ARs blood concentrations above $80 \mathrm{nM}$ (>99 $\mathrm{nM}$ in men and $>84 \mathrm{nM}$ ), the IRR of distal colon cancer was reduced by $52 \%$ (78 cases, 114 controls). Moreover, a $17 \%$ reduction in IRR of colon cancer was reported for Scandinavian participants (252 case-control pairs). The authors thus indicate an inverse correlation between the total concentrations of ARs in plasma and the risk of colon and distal colon cancer development, although the phenomenon was observed only in those individuals who come from areas with high, stable, and frequent ARs intake, namely, Central Europe and Scandinavia.

Another study of the AR-colorectal cancer relationship, conducted with the participation of approximately one hundred twenty thousand men and women aged 30-64 years, reported a $64 \%$ decreased risk of distal colon cancer (198 cases-control pairs) in individuals with higher ARs plasma concentrations $(>118.6 \mathrm{nM}$ for men and $>91.7 \mathrm{nM}$ for women) compared to those with lower ARs levels in blood ( $\leq 35.3 \mathrm{nM}$ for men and $<27.5 \mathrm{nM}$ for women) [81].

Anticancer properties of ARs are being characterized in multiple in vitro studies using human hepatocarcinoma, ovarian, cervical, colon, lung, central nervous system, and breast cancer cell lines as models, and some of the results to date are perfectly observed in the review of Kruk et al. [1]. In brief, ARs are able to inhibit the growth of cancer cells in the human breast, lung, and central nervous system. In the study of Sanchez et al. [82], they tested the cytotoxicity of five isolated natural ARs on MCF-7, H-460, and SF-268 cell lines and demonstrated the $800 \%$ stronger inhibitory effect of ARs over the commonly known cytostatic agent Adriamycin. The results of this study are in concordance with the previously reported antiproliferative effect of 14 different ARs isolated from the leaves of a tropical ornamental tree applied to human breast carcinoma, lung carcinoma, and central nervous system carcinoma cells [83].

Two human hepatocarcinoma cell lines, HepG2 and Hep3B, were used to demonstrate the cytotoxic effect of 5alkylresorcinol (5-AR) from the leaves of L. molleoides, and in both of them 24-hour exposure to 5-AR resulted in the fragmentation of DNA and the nuclear condensation, characteristic for apoptotic cells [84]. Interestingly, the cell death induction in the cultures occurred in a p53-independent way. Other investigations of the last three decades confirm the property of ARs to inhibit the growth of tumor cells, particularly, ovarian cancer cells [85], human prostate adenocarcinoma (PC3) cells [69], breast cancer cell line $[82,83,86]$, and cervical cancer (HeLa) cell line [87]. Taken together, these facts propose that ARs can be promising adjuvants in anticancer therapy.

The exact mechanisms of ARs anticancer properties remain unclear due to multiple ARs targets and the complexity of interrelations between various biochemical actions. Apparently, ARs affect cellular morphology via the fragmentation of DNA and the condensation of nuclei leading to apoptosis [84], in particular, in cells already damaged by genotoxic agents [71]. At the same time, other researchers report the antioxidant properties of ARs, which manifest in the reduction of the amount of ROS and/or in the inhibition of the enzymes involved in the production of free radicals under physiological conditions [88]. Although the anticancer properties of ARs are obvious, further studies are needed to elucidate the exact molecular mechanisms of ARs action.

5.9. ARs and Metabolic Health. It is generally accepted that Mediterranean diet, which consists of a lot of fruits, vegetables, legumes, nuts, and whole grain products, is beneficial for human metabolic health. The advantages of such a diet are not only in getting an appropriate amount of vitamins, microelements, and antioxidant agents, but also in the consumption of such regulatory substances like ARs. As it was shown by Rejman et al. on 3T3-L1 cell culture, ARs prevented the cytoplasmic accumulation of triglycerides, possibly by the in vivo sirtuin-dependent inhibition of triglyceride synthesis [89]. Negative correlation between the level of alkylresorcinols and nonesterified fatty acids concentrations in human plasma samples was demonstrated after the switch to a whole grain diet [90]. Additionally, ARs were shown to inhibit lipolysis carried out by hormonesensitive lipase [91] and to block the activity of glycerol-3phosphate dehydrogenase, which is in charge of triglyceride accumulation [52]. The addition of solely ARs to the "high fat/high sucrose (FS) diet» managed not only to suppress the accumulation of triglycerides in the liver of mice, but also to overcome hyperinsulinemia and hyperleptinemia in the FS group of animals [46]. Other stunning results of this experiment include the marked ARs-dependent reduction of fasting blood glucose concentrations and the suppression of both glucose intolerance and insulin resistance induced by the FS diet. Oishi et al. also found that ARs significantly enhanced insulin-stimulated hepatic Akt phosphorylation, which might be associated with the increased irs gene expression in the liver. Besides, circulating plasma total cholesterol level decreased along with the increased fecal cholesterol excretion, which clearly contributes to the ARsmediated suppression of the accumulation of lipids in the liver as well as the overcoming of glucose intolerance and insulin resistance. Thus, ARs successfully prevented dietinduced obesity in mice, and it is supported by the fact that, despite the similar daily caloric intake, mice fed with FS diet gained more weight compared to the group provided with ARs along with the FS ration. According to the study, ARs significantly suppressed the FS-induced increase in plasma leptin concentration and concomitant adipose mRNA expression. Since leptin is known as one of the major satiety signaling molecules, which travel from adipose tissue to the hypothalamic centers that regulate appetite [92], ARs might suppress leptin resistance in the hypothalamus. However, according to the investigation of Oishi et al., ARs did not affect either gluconeogenesis in the liver, or the expression of genes associated with gluconeogenesis, such asG6pc and Pck1, but significantly enhanced the expression of Irs 1 


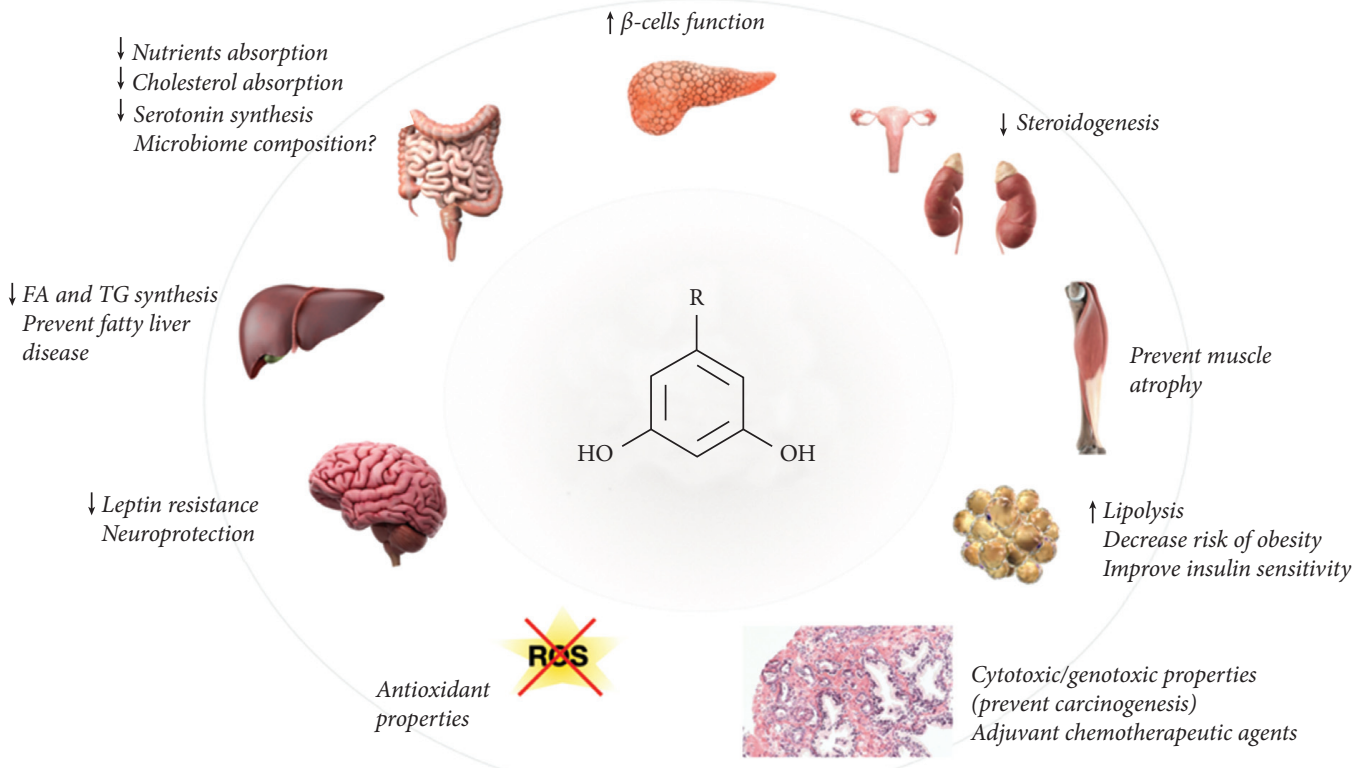

FIgURE 5: The main regulatory functions of ARs according to in vivo and in vitro studies.

mRNA during the feeding period compared to the FS diet, and this corresponded to Akt phosphorylation [46]. Undoubtedly, deeper studies are required to reveal the underlying mechanisms of the AR-induced increase in hepatic insulin sensitivity.

The results of the study described above are well consistent with the research of Malin et al., in which they showed the significant improvement in the glucose-stimulated insulin secretion (GSIS) response in middle-aged overweight/obese adults at risk for type 2 diabetes, who followed a whole grain diet compared to those provided with refined grains [93]. Thus, the inclusion of whole grains in a daily ration may help an individual in the compensation for insulin resistance due to $\beta$-cell stimulation and additional activation of insulin secretion. Such an approach could serve to prevent the progression to diabetes in an at-risk obese population with prediabetes. The authors suggested several mechanisms by which whole grains may increase GSIS. First, the diet rich in whole grains promotes weight loss due to the reduction of fat mass [94], which, in its turn, can lower the blood concentrations of lipids and/or inflammatory factors known to disturb $\beta$-cell insulin secretion [95]). Second, gut hormones from the family of incretins, such as GLP-1 (glucagon-like peptide 1) and GIP (gastric inhibitory polypeptide), which are secreted in response to nutrient consumption [96], are hypothesized to be elevated together with insulin in the individuals provided with whole grain products. However, according to the observations of Malin et al., whole grain intake had no effect on gut hormones compared to refined grains, and both whole grain and refined grain interventions reduced body weight and fat loss [93].

Diet enriched in whole grains is associated with lower concentrations of serotonin, taurine, and glycerophosphocholine in blood [97]. Martin at al. demonstrated the decreased colonic serotonin synthesis in mice fed with rye bran compared to those supported with cellulose as dietary fiber [98]. Although gut-derived serotonin is an important mediator in normal gastrointestinal physiology, the inhibition of its synthesis is evidenced to reduce obesity and metabolic dysfunction and ameliorate type 2 diabetes [99]. Since elevated blood serotonin is linked to the reduced glycemic control, the findings of the whole grain-induced decrease in serotonin production are intriguing. At the same time, the influence of ARs on the synthesis of serotonin in the intestine, as well as their role in the improvement of sensitivity to insulin and the reduction of the risk of type 2 diabetes development, remains to be elucidated.

As it was mentioned previously, ARs consumption may decrease body weight by inhibiting nutrient absorption. ARs contain hydrophobic alkyl chains able to react with human digestive enzymes such as $\alpha$-glucosidase, trypsin, and aldose reductase, thus affecting carbohydrate digestion [22]. Additionally, Song et al. in their research of $\alpha$-glucosidase kinetics managed to demonstrate the 4-hexylresorcinol potential as a noncompetitive reversible inhibitor of the enzyme. The substance tested also presented the ability to interfere with nonenzymatic glycation reactions, thus reducing the formation of fructosamines and blocking the synthesis of $\alpha$-dicarbonyl compounds and advanced glycation end products (AGEs) [100]. In vitro investigations confirmed ARs inhibitory effects on $\alpha$-glucosidase [101]. Based on these data, 4-hexylresorcinol can be considered as a possible preventive and therapeutic agent for adult-onset diabetes.

Another beneficial effect of a whole grain diet is a lower risk of fatty liver, although the underlying mechanisms remain unknown [102]. Nonalcoholic hepatosteatosis is closely associated with insulin resistance and the promotion of impaired glucose tolerance [103]. In vivo studies in mice demonstrated the significant ARs-dependent suppression of hepatic triglyceride accumulation induced by fat-rich 
diet, although neither the concentrations of circulating free fatty acids, nor the mRNA expression of both lipogenicand $\beta$-oxidation-related genes were affected [46]. Besides, ARs were shown to increase the fecal excretion of total lipids and cholesterol and to significantly reduce the amounts of fecal bile acids, although the effect was strong only in conditions of elevated hepatic bile secretion. These findings suggest that the increased fecal cholesterol excretion was independent of the increased excretion of fecal bile acids and most likely linked to the ability of ARs as hydrophobic compounds, to reduce the capacity to carry micellar lipids by binding bile acids and suppressing intestinal cholesterol absorption, although the precise mechanism remains to be clarified [46].

\section{Conclusions}

This systematic review is aimed at the summary of the beneficial effects of ARs on human health. Plenty of evidence indicating diverse ARs bioactivities has been accumulated in recent years (Figure 5).

In vivo and in vitro studies, as well as epidemiological investigations, have shown that ARs can affect many physiological and pathological processes related to the immune system, metabolic regulation, cell signaling, and gene expression regulation. Currently, ARs are used as biomarkers of whole grain wheat and rye product consumption and are proposed to be utilized as adjuvant anticancer and antimicrobial therapeutic agents. Due to ARs ability to take on the role of autoregulators of microbial activity, further investigations concerning their role in "gut microbiomehost organism» interactions are needed.

There are some observations concerning ARs neuroprotective, muscle-protective, and metabolic improving effects. Strong evidence was received about the positive influence of a whole grain diet and increased levels of ARs on human metabolic health.

High plasma concentrations of ARs are proved to be associated with the reduced risk of colon, prostate, and breast cancers. Numerous in vitro studies report the highly cytotoxic properties of high concentrations of ARs for certain types of cancer, particularly, human colon, breast, lung, central nervous, ovarian, cervical, and prostate tumors, and hepatocarcinoma cancer cell lines. Furthermore, the phenolic ring and alkyl chain as structural components of ARs are important for the inhibition of human cancer cell proliferation, although the exact mechanisms of the toxicity are not clear to date.

There are some hypothesized molecular mechanisms of ARs action as bioregulators, including their ability to affect all enzyme-regulated processes in cells, suppress lipolysis in adipocytes, cause genotoxicity, insert into the membranes of erythrocytes, and exert indirect antioxidant capacity.

The characterization of ARs properties provides further support for public health recommendations emphasizing diets rich in whole grains as potentially preventive against metabolic, immune, oncological, and other chronic diseases.

\section{Conflicts of Interest}

The authors declare that they have no conflicts of interest.

\section{References}

[1] B. J. Kruk, Aboul-Enein, and J. Berstein, "Dietary alkylresorcinols and cancer prevention: a systematic review," European Food Research and Technology, vol. 243, pp. 1693-1710, 2021.

[2] G. Zong, B. Lebwohl, F. B. Hu et al., "Gluten intake and risk of type 2 diabetes in three large prospective cohort studies of US men and women," Diabetologia, vol. 61, no. 10, pp. 2164-2173, 2018.

[3] M. G. Rooks and W. S. Garrett, "Gut microbiota, metabolites and host immunity," Nature Reviews Immunology, vol. 16, no. 6, pp. 341-352, 2016.

[4] H. Xiao and S. Kang, "The role of the gut microbiome in energy balance with a focus on the gut-adipose tissue Axis," Frontiers in Genetics, vol. 11, p. 297, 2020.

[5] J. F. Cryan, K. J. O'Riordan, C. S. M. Cowan et al., "The microbiota-gut-brain Axis," Physiological Reviews, vol. 99, no. 4, pp. 1877-2013, 2019.

[6] T. C. Fung, C. A. Olson, and E. Y. Hsiao, "Interactions between the microbiota, immune and nervous systems in health and disease," Nature Neuroscience, vol. 20, no. 2, pp. 145-155, 2017.

[7] M. B. Austin and J. P. Noel, "The chalcone synthase superfamily of type III polyketide synthases," Natural Product Reports, vol. 20, no. 1, pp. 79-110, 2003.

[8] M. Funabashi, N. Funa, and S. Horinouchi, "Phenolic lipids synthesized by type III polyketide synthase confer penicillin resistance on Streptomyces griseus," Journal of Biological Chemistry, vol. 283, no. 20, pp. 13983-13991, 2008.

[9] A. Miyanaga and S. Horinouchi, "Enzymatic synthesis of bis5-alkylresorcinols by resorcinol-producing type III polyketide synthases," Journal of Antibiotics, vol. 62, no. 7, pp. 371-376, 2009.

[10] M. Matsuzawa, Y. Katsuyama, N. Funa, and S. Horinouchi, "Alkylresorcylic acid synthesis by type III polyketide synthases from rice Oryza sativa," Phytochemistry, vol. 71, no. 10, pp. 1059-1067, 2010.

[11] Y. Zhu, D. R. Conklin, H. Chen, L. Wang, and S. Sang, "5alk(en)ylresorcinols as the major active components in wheat bran inhibit human colon cancer cell growth," Bioorganic \& Medicinal Chemistry, vol. 19, no. 13, pp. 3973-3982, 2011.

[12] A. Kozubek and J. H. P. Tyman, "Resorcinolic lipids, the natural non-isoprenoid phenolic amphiphiles and their biological activity," Chemical Reviews, vol. 99, no. 1, pp. 1-26, 1999.

[13] Y. Zhu and S. Sang, "Phytochemicals in whole grain wheat and their health-promoting effects," Molecular Nutrition \& Food Research, vol. 61, no. 7, 2017.

[14] Y. S. Kim, D. W. Kim, S. G. Kim, and S. K. Lee, "4-hexylresorcinol-induced protein expression changes in human umbilical cord vein endothelial cells as determined by immunoprecipitation high-performance liquid chromatography," PLoS One, vol. 15, no. 12, Article ID e0243975, 2020.

[15] N. Tsuge, M. Mizokami, S. Imai, A. Shimazu, and H. Seto, "Adipostatins A and B, new inhibitors of glycerol-3-phosphate dehydrogenase," Journal of Antibiotics, vol. 45, no. 6, pp. 886-891, 1992.

[16] A. Kozubek, S. Pietr, and A. Czerwonka, "Alkylresorcinols are abundant lipid components in different strains of 
Azotobacter chroococcum and Pseudomonas spp," Journal of Bacteriology, vol. 178, no. 14, pp. 4027-4030, 1996.

[17] T. Hayashi, Y. Kitamura, N. Funa, Y. Ohnishi, and S. Horinouchi, "Fatty acyl-AMP ligase involvement in the production of alkylresorcylic acid by a Myxococcus xanthus type III polyketide synthase," ChemBioChem, vol. 12, no. 14, pp. 2166-2176, 2011.

[18] T. P. Martins, C. Rouger, N. R. Glasser et al., "Chemistry, bioactivity and biosynthesis of cyanobacterial alkylresorcinols," Natural Product Reports, vol. 36, no. 10, pp. 1437-1461, 2019.

[19] M. Stasiuk and A. Kozubek, "Biological activity of phenolic lipids," Cellular and Molecular Life Sciences, vol. 67, no. 6, pp. 841-860, 2010.

[20] M. Marklund, E. A. Strömberg, H. N. Lærke et al., "Simultaneous pharmacokinetic modeling of alkylresorcinols and their main metabolites indicates dual absorption mechanisms and enterohepatic elimination in humans," Journal of Nutrition, vol. 144, no. 11, pp. 1674-1680, 2014.

[21] R. Landberg, P. Åman, L. E. Friberg, B. Vessby, H. Adlercreutz, and A. Kamal-Eldin, "Dose response of whole-grain biomarkers: alkylresorcinols in human plasma and their metabolites in urine in relation to intake," The American Journal of Clinical Nutrition, vol. 89, no. 1, pp. 290-296, 2009.

[22] A. B. Ross, Y. Chen, J. Frank et al., "Cereal alkylresorcinols elevate $\gamma$-tocopherol levels in rats and inhibit $\gamma$-tocopherol metabolism in vitro," Journal of Nutrition, vol. 134, no. 3 , pp. 506-510, 2004a.

[23] A. B. Ross, A. Kamal-Eldin, and P. Åman, "Dietary alkylresorcinols: absorption, bioactivities, and possible use as biomarkers of whole-grain wheat- and rye-rich foods," Nutrition Reviews, vol. 62, no. 3, pp. 81-95, 2004 b.

[24] H. M. Roager, J. K. Vogt, M. Kristensen et al., "Whole grainrich diet reduces body weight and systemic low-grade inflammation without inducing major changes of the gut microbiome: a randomised cross-over trial," Gut, vol. 68, no. 1, pp. 83-93, 2019.

[25] R. Landberg, R. Wierzbicka, L. Shi et al., "New alkylresorcinol metabolites in spot urine as biomarkers of whole grain wheat and rye intake in a Swedish middle-aged population," European Journal of Clinical Nutrition, vol. 72, no. 10, pp. 1439-1446, 2018.

[26] A. Rigotti, "Absorption, transport, and tissue delivery of vitamin E," Molecular Aspects of Medicine, vol. 28, no. 5-6, pp. 423-436, 2007.

[27] A. M. Linko-Parvinen, R. Landberg, M. J. Tikkanen, H. Adlercreutz, and J. L. Penalvo, "Alkylresorcinols from whole-grain wheat and rye are transported in human plasma lipoproteins," Journal of Nutrition, vol. 137, no. 5, pp. 1137-1142, 2007.

[28] A. Kozubek, "The effect of 5-(n-alk(en)yl)resorcinols on membranes. II. Dependence of the aliphatic chain length and unsaturation," Acta Biochimica Polonica, vol. 34, no. 4, pp. 387-394, 1987.

[29] M. V. Lind, M. L. Madsen, J. J. Rumessen et al., "Plasma alkylresorcinols reflect gluten intake and distinguish between gluten-rich and gluten-poor diets in a population at risk of metabolic syndrome," Journal of Nutrition, vol. 146, no. 10, pp. 1991-1998, 2016.

[30] Y. Zhu, K. L. Shurlknight, X. Chen, and S. Sang, "Identification and pharmacokinetics of novel alkylresorcinol metabolites in human urine, new candidate biomarkers for whole-grain wheat and rye intake," Journal of Nutrition, vol. 144, no. 2, pp. 114-122, 2014.
[31] N. M. McKeown, M. Marklund, J. Ma et al., "Comparison of plasma alkylresorcinols (AR) and urinary AR metabolites as biomarkers of compliance in a short-term, whole-grain intervention study," European Journal of Nutrition, vol. 55, no. 3, pp. 1235-1244, 2016.

[32] B. S. Lynch, E. S. Delzell, and D. H. Bechtel, "Toxicology review and risk assessment of resorcinol: thyroid effects," Regulatory Toxicology and Pharmacology, vol. 36, no. 2, pp. 198-210, 2002.

[33] A. M. Linko, K. Parikka, K. Wahala, and H. Adlercreutz, "Gas chromatographic-mass spectrometric method for the determination of alkylresorcinols in human plasma," Analytical Biochemistry, vol. 308, no. 2, pp. 307-313, 2001.

[34] Y. A. Nikolaev, A. V. Tutel'yan, N. G. Loiko et al., "The use of 4-Hexylresorcinol as antibiotic adjuvant," PLoS One, vol. 15, no. 9, Article ID e0239147, 2020.

[35] N. Q. Balaban, J. Merrin, R. Chait, L. Kowalik, and S. Leibler, "Bacterial persistence as a phenotypic switch," Science, vol. 305, no. 5690, pp. 1622-1625, 2004.

[36] B. Van den Bergh, J. E. Michiels, T. Wenseleers et al., "Frequency of antibiotic application drives rapid evolutionary adaptation of Escherichia coli persistence," Nature Microbiology, vol. 1, Article ID 16020, 2016.

[37] S. Helaine and E. Kugelberg, "Bacterial persisters: formation, eradication, and experimental systems," Trends in Microbiology, vol. 22, no. 7, pp. 417-424, 2014.

[38] O. V. Bukharin, N. B. Perunova, G. I. El-Registan et al., "Influence of chemical analogue of extracellular microbial autoregulators on antilysozyme activity of bacteria," Zhurnal Mikrobiologii, Epidemiologii I Immunobiologii, vol. 6, pp. 3-6, 2007.

[39] G. I. El'-Registan, A. L. Muliukin, I. Nikolaev, N. E. Suzina, V. F. Gal'chenko, and V. I. Duda, "Adaptive functions of extracellular autoregulators of microorganisms," Mikrobiologiia, vol. 75, no. 4, pp. 446-456, 2006.

[40] I. A. Nikolaev, A. L. Muliukin, I. I. Stepanenko, and G. I. El'-Registan, "Autoregulation of stress response in microorganisms," Mikrobiologiia, vol. 75 , no. 4, pp. 489-496, 2006.

[41] D. G. Deryabian, N. A. Romanenko, and G. I. El-Registan, "Change of the specificity of antibodies in the presence of alkylphenylalkohol, chemical analog of bacterial autoregulation molecules," Immunologiya, vol. 34, no. 1, pp. 2730, 2021, in Russian.

[42] L. M. Cox and M. J. Blaser, "Pathways in microbe-induced obesity," Cell Metabolism, vol. 17, no. 6, pp. 883-894, 2013.

[43] V. Tremaroli and F. Backhed, "Functional interactions between the gut microbiota and host metabolism," Nature, vol. 489, no. 7415, pp. 242-249, 2012.

[44] P. Kovatcheva-Datchary and T. Arora, "Nutrition, the gut microbiome and the metabolic syndrome," Best Practice of Research Clinics in Gastroenterology, vol. 27, no. 1, pp. 59-72, 2013.

[45] A. Costabile, A. Klinder, F. Fava et al., "Whole-grain wheat breakfast cereal has a prebiotic effect on the human gut microbiota: a double-blind, placebo-controlled, crossover study," British Journal of Nutrition, vol. 99, no. 1, pp. 110-120, 2008.

[46] K. Oishi, S. Yamamoto, N. Itoh et al., "Wheat alkylresorcinols suppress high-fat, high-sucrose diet-induced obesity and glucose intolerance by increasing insulin sensitivity and cholesterol excretion in male mice," Journal of Nutrition, vol. 145, no. 2, pp. 199-206, 2015. 
[47] U. Etxeberria, A. Fernandez-Quintela, F. I. Milagro, L. Aguirre, J. A. Martinez, and M. P. Portillo, "Impact of polyphenols and polyphenol-rich dietary sources on gut microbiota composition," Journal of Agricultural and Food Chemistry, vol. 61, no. 40, pp. 9517-9533, 2013.

[48] S. Golbidi, A. Daiber, B. Korac, H. Li, M. F. Essop, and I. Laher, "Health benefits of fasting and caloric restriction," Current Diabetes Reports, vol. 17, no. 12, p. 123, 2017.

[49] Y. Jia, Z. Zheng, Y. Wang et al., "SIRT1 is a regulator in high glucose-induced inflammatory response in RAW264.7 cells," PLoS One, vol. 10, no. 3, Article ID e0120849, 2015.

[50] H. C. Chang and L. Guarente, "SIRT1 and other sirtuins in metabolism," Trends in Endocrinology and Metabolism, vol. 25, no. 3, pp. 138-145, 2014.

[51] Y. Kayashima, Y. Katayanagi, K. Tanaka, R. Fukutomi, S. Hiramoto, and S. Imai, "Alkylresorcinols activate SIRT1 and delay ageing in Drosophila melanogaster," Scientific Reports, vol. 7, p. 43679, 2017.

[52] J. Rejman, "Long-chain orcinol homologs from cereal bran are effective inhibitors of glycerophosphate dehydrogenase," Cellular and Molecular Biology Letters, vol. 2, pp. 411-419, 1997.

[53] R. T. Evans, P. J. Baker, R. A. Coburn, S. L. Fischman, and R. J. Genco, "In vitro antiplaque effects of antiseptic phenols," Journal of Periodontology, vol. 48, no. 3, pp. 156-162, 1977.

[54] D. McNally, A. Shephard, and E. Field, "Randomised, double-blind, placebo-controlled study of a single dose of an amylmetacresol/2,4-dichlorobenzyl alcohol plus lidocaine lozenge or a hexylresorcinol lozenge for the treatment of acute sore throat due to upper respiratory tract infection," Journal of Pharmacy \& Pharmaceutical Sciences, vol. 15, no. 2, pp. 281-294, 2012.

[55] J. Ahn, S. G. Kim, M. K. Kim et al., "Topical delivery of 4hexylresorcinol promotes wound healing via tumor necrosis factor-alpha suppression," Burns, vol. 42, no. 7, pp. 1534-1541, 2016.

[56] P. Farris, J. Zeichner, and D. Berson, "Efficacy and tolerability of a skin brightening/anti-aging cosmeceutical containing retinol $0.5 \%$, niacinamide, hexylresorcinol, and resveratrol," Journal of Drugs in Dermatology, vol. 15, no. 7, pp. 863-868, 2016.

[57] Z. D. Draelos, I. Diaz, A. Cohen, J. Mao, and T. Boyd, “A novel skin brightening topical technology," Journal of Cosmetic Dermatology, vol. 19, no. 12, pp. 3280-3285, 2020.

[58] M. A. Ruegg and D. J. Glass, "Molecular mechanisms and treatment options for muscle wasting diseases," Annual Review of Pharmacology and Toxicology, vol. 51, pp. 373-395, 2011.

[59] S. Hiramoto, N. Yahata, K. Saitoh et al., "Dietary supplementation with alkylresorcinols prevents muscle atrophy through a shift of energy supply," The Journal of Nutritional Biochemistry, vol. 61, pp. 147-154, 2018.

[60] A. Trombetti, K. F. Reid, M. Hars et al., "Age-associated declines in muscle mass, strength, power, and physical performance: impact on fear of falling and quality of life," Osteoporosis International, vol. 27, no. 2, pp. 463-471, 2016.

[61] R. L. Marcus, O. Addison, J. P. Kidde, L. E. Dibble, and P. C. Lastayo, "Skeletal muscle fat infiltration: impact of age, inactivity, and exercise," The Journal of Nutrition, Health \& Aging, vol. 14, no. 5, pp. 362-366, 2010.
[62] B. N. Dugger and D. W. Dickson, "Pathology of neurodegenerative diseases," Cold Spring Harbor Perspectives in Biology, vol. 9, no. 7, Article ID a028035, 2017.

[63] M. Koryciniska, K. Czelna, A. Jaromin, and A. Kozubek, "Antioxidant activity of rye bran alkylresorcinols and extracts from whole-grain cereal products," Food Chemistry, vol. 116, pp. 1013-1018, 2009.

[64] F. Fan, Y. Zou, Y. Fang et al., "Potential neuroprotection of wheat alkylresorcinols in hippocampal neurons via Nrf2/ ARE pathway," Food \& Function, vol. 11, no. 11, pp. 10161-10169, 2020.

[65] B. Opitz, "Memory function and the hippocampus," Frontiers of Neurology and Neuroscience, vol. 34, pp. 51-59, 2014.

[66] J. Liu, L. Li, and W. Z. Suo, "HT22 hippocampal neuronal cell line possesses functional cholinergic properties," Life Sciences, vol. 84, no. 9-10, pp. 267-271, 2009.

[67] L. Yuan, J. Liu, J. Zhen, C. Cai, S. Wang, and R. Xiao, "Serum collected from fruit and vegetable juice treated rats antagonizing $\mathrm{H}_{2} \mathrm{O}_{2}$-induced oxidative damage in PC12 cells," Journal of Functional Foods, vol. 20, pp. 496-505, 2016.

[68] H. E. de Vries, M. Witte, D. Hondius et al., "Nrf2-induced antioxidant protection: a promising target to counteract ROS-mediated damage in neurodegenerative disease?" Free Radical Biology \& Medicine, vol. 45, no. 10, pp. 1375-1383, 2008.

[69] L. Liu, K. M. Winter, L. Stevenson, C. Morris, and D. N. Leach, "Wheat bran lipophilic compounds with in vitro anticancer effects," Food Chemistry, vol. 130, pp. 156-164, 2012.

[70] S. R. Starck, J. Z. Deng, and S. M. Hecht, "Naturally occurring alkylresorcinols that mediate DNA damage and inhibit its repair," Biochemistry, vol. 39, no. 9, pp. 2413-2419, 2000.

[71] K. Gasiorowski, B. Brokos, A. Kulma, A. Ogorzalek, and K. Skorkowska, "Impact of four antimutagens on apoptosis in genotoxically damaged lymphocytes in vitro," Cellular and Molecular Biology Letters, vol. 6, no. 3, pp. 649-675, 2001.

[72] A. Oskarsson and A. A. Ohlsson, "Suppressed sex hormone biosynthesis by alkylresorcinols: a possible link to chemoprevention," Nutrition and Cancer, vol. 68, no. 6, pp. 978-987, 2016.

[73] A. Gsur, G. Bernhofer, S. Hinteregger et al., "A polymorphism in the CYP17 gene is associated with prostate cancer risk," International Journal of Cancer, vol. 87, no. 3, pp. 434-437, 2000.

[74] J. E. Torfadottir, U. A. Valdimarsdottir, L. Mucci et al., "Rye bread consumption in early life and reduced risk of advanced prostate cancer," Cancer Causes \& Control, vol. 23, no. 6, pp. 941-950, 2012.

[75] I. Drake, E. Sonestedt, B. Gullberg et al., "Plasma alkylresorcinol metabolites as biomarkers for whole-grain intake and their association with prostate cancer: a Swedish nested case-control study," Cancer Epidemiology, Biomarkers \& Prevention: A Publication of the American Association for Cancer Research, vol. 23, no. 1, pp. 73-83, 2014.

[76] A. Subramanian, M. Salhab, and K. Mokbel, "Oestrogen producing enzymes and mammary carcinogenesis: a review," Breast Cancer Research and Treatment, vol. 111, no. 2, pp. 191-202, 2008.

[77] H. Adlercreutz, "Can rye intake decrease risk of human breast cancer?" Food \& Nutrition Research, vol. 54, 2010.

[78] D. Aune, D. S. Chan, D. C. Greenwood et al., "Dietary fiber and breast cancer risk: a systematic review and meta-analysis of prospective studies," Annals of Oncology, vol. 23, no. 6, pp. 1394-1402, 2012. 
[79] P. Ferrari, S. Rinaldi, M. Jenab et al., "Dietary fiber intake and risk of hormonal receptor-defined breast cancer in the $\mathrm{Eu}$ ropean Prospective Investigation into Cancer and Nutrition study," The American Journal of Clinical Nutrition, vol. 97, no. 2, pp. 344-353, 2013.

[80] C. Kyro, A. Olsen, R. Landberg et al., "Plasma alkylresorcinols, biomarkers of whole-grain wheat and rye intake, and incidence of colorectal cancer," Journal of the National Cancer Instute, vol. 106, no. 1, Article ID djt352, 2014.

[81] M. D. Knudsen, C. Kyro, A. Olsen et al., "Self-reported whole-grain intake and plasma alkylresorcinol concentrations in combination in relation to the incidence of colorectal cancer," American Journal of Epidemiology, vol. 179, no. 10, pp. 1188-1196, 2014.

[82] L. A. Sanchez, D. Olmedo, J. L. Lopez-Perez, T. D. Williams, and M. P. Gupta, "Two new alkylresorcinols from Homalomena wendlandii and their cytotoxic activity," Natural Product Communications, vol. 7, no. 8, pp. 1043-1046, 2012.

[83] T. H. Chuang and P. L. Wu, "Cytotoxic 5-alkylresorcinol metabolites from the leaves of Grevillea robusta," Journal of Natural Products, vol. 70, no. 2, pp. 319-323, 2007.

[84] L. Barbini, P. Lopez, J. Ruffa et al., "Induction of apoptosis on human hepatocarcinoma cell lines by an alkyl resorcinol isolated from Lithraea molleoides," World Journal of Gastroenterology, vol. 12, no. 37, pp. 5959-5963, 2006.

[85] V. S. Chaturvedula, J. K. Schilling, J. S. Miller, R. Andriantsiferana, V. E. Rasamison, and D. G. Kingston, "New cytotoxic bis 5-alkylresorcinol derivatives from the leaves of Oncostemon bojerianum from the Madagascar rainforest," Journal of Natural Products, vol. 65, no. 11, pp. 1627-1632, 2002.

[86] N. A. Al-Mekhlafi, K. Shaari, F. Abas et al., "Alkenylresorcinols and cytotoxic activity of the constituents isolated from Labisia pumila," Phytochemistry, vol. 80, pp. 42-49, 2012.

[87] I. Kubo, M. Ochi, P. C. Vieira, and S. Komatsu, "Antitumor agents from the cashew (Anacardium occidentale) apple juice," Journal of Agricultural and Food Chemistry, vol. 41, pp. 1012-1015, 1993.

[88] A. Luis, F. Domingues, and A. P. Duarte, "Biological properties of plant-derived alkylresorcinols," Mini Reviews in Medicinal Chemistry, vol. 16, no. 11, pp. 851-854, 2016.

[89] J. Rejman and A. Kozubek, "Inhibitory effect of natural phenolic lipids upon NAD-dependent dehydrogenases and on triglyceride accumulation in 3T3-L1 cells in culture," Journal of Agricultural and Food Chemistry, vol. 52, no. 2, pp. 246-250, 2004.

[90] A. B. Ross, A. Bourgeois, H. N. Macharia et al., "Plasma alkylresorcinols as a biomarker of whole-grain food consumption in a large population: results from the WHOLEheart Intervention Study," The American Journal of Clinical Nutrition, vol. 95, no. 1, pp. 204-211, 2012.

[91] U. Andersson, E. S. Dey, C. Holm, and E. Degerman, "Rye bran alkylresorcinols suppress adipocyte lipolysis and hormone-sensitive lipase activity," Molecular Nutrition \& Food Research, vol. 55, no. 2, pp. 290-293, 2011.

[92] G. A. Bray, "Afferent signals regulating food intake," Proceedings of the Nutrition Society, vol. 59, no. 3, pp. 373-384, 2000.

[93] S. K. Malin, E. L. Kullman, A. R. Scelsi, J. P. Godin, A. B. Ross, and J. P. Kirwan, "A whole-grain diet increases glucose-stimulated insulin secretion independent of gut hormones in adults at risk for type 2 diabetes," Molecular
Nutrition \& Food Research, vol. 63, no. 7, Article ID e1800967, 2019.

[94] J. P. Karl, M. Meydani, J. B. Barnett et al., "Substituting whole grains for refined grains in a 6-wk randomized trial favorably affects energy-balance metrics in healthy men and postmenopausal women," The American Journal of Clinical Nutrition, vol. 105, no. 3, pp. 589-599, 2017.

[95] K. M. Utzschneider, R. L. Prigeon, M. V. Faulenbach et al., "Oral disposition index predicts the development of future diabetes above and beyond fasting and 2-h glucose levels," Diabetes Care, vol. 32, no. 2, pp. 335-341, 2009.

[96] I. Lee, L. Shi, D. L. Webb, P. M. Hellstrom, U. Riserus, and R. Landberg, "Effects of whole-grain rye porridge with added inulin and wheat gluten on appetite, gut fermentation and postprandial glucose metabolism: a randomised, cross-over, breakfast study," British Journal of Nutrition, vol. 116, no. 12, pp. 2139-2149, 2016.

[97] P. Keski-Rahkonen, M. Kolehmainen, J. Lappi et al., "Decreased plasma serotonin and other metabolite changes in healthy adults after consumption of whole grain rye: an untargeted metabolomics study," The American Journal of Clinical Nutrition, vol. 109, no. 6, pp. 1630-1639, 2019.

[98] A. M. Martin, R. L. Young, L. Leong et al., "The diverse metabolic roles of peripheral serotonin," Endocrinology, vol. 158, no. 5, pp. 1049-1063, 2017.

[99] J. D. Crane, R. Palanivel, E. P. Mottillo et al., "Inhibiting peripheral serotonin synthesis reduces obesity and metabolic dysfunction by promoting brown adipose tissue thermogenesis," Nature Medicine, vol. 21, no. 2, pp. 166-172, 2015.

[100] S. Song, Q. Liu, W. M. Chai, S. S. Xia, Z. Y. Yu, and Q. M. Wei, "Inhibitory potential of 4-hexylresorcinol against alpha-glucosidase and non-enzymatic glycation: activity and mechanism," Journal of Bioscience and Bioengenering, vol. 131, no. 3, pp. 241-249, 2021.

[101] J. Tu, J. Chen, S. Zhu, C. Zhang, H. Chen, and Y. Liu, "Inhibition of wheat bran and it's active components on alpha-glucosidase in vitro," Pharmacognosy Magazine, vol. 9, no. 36, pp. 309-314, 2013.

[102] A. B. Ross, J. P. Godin, K. Minehira, and J. P. Kirwan, "Increasing whole grain intake as part of prevention and treatment of nonalcoholic Fatty liver disease," International Journal of Endocrinology, vol. 2013, Article ID 585876, 13 pages, 2013.

[103] T. Takamura, H. Misu, T. Ota, and S. Kaneko, "Fatty liver as a consequence and cause of insulin resistance: lessons from type 2 diabetic liver," Endocrine Journal, vol. 59, no. 9, pp. 745-763, 2012. 